\title{
TRANSIENT IGNITION AND COMBUSTION OF DILUTED HYDROGEN/AIR MIXTURES BY A THIN CATALYTIC WIRE
}

\author{
C. TREVIÑO ${ }^{1,2}$ F. J. HIGUERA ${ }^{2}$ AND A. LIÑÁN ${ }^{2}$ \\ ${ }^{1}$ Facultad de Ciencias \\ UNAM \\ 04510 Mexico D. F., Mexico \\ ${ }^{2}$ ETSI Aeronáuticos \\ $U P M$ \\ Plaza del Cardenal Cisneros 3 \\ 28040 Madrid, Spain
}

\begin{abstract}
This paper reports on a study of transient ignition and combustion of hydrogen/air mixtures by a heated, thin catalytic wire in a natural convection environment. Modeling of the process is accomplished via a reduced set of heterogeneous kinetic processes which include dissociative adsorption and desorption of both reactants, three fast surface reactions of the Langmiur-Hinshelwood type, and the desorption reaction of the adsorbed product, $\mathrm{H}_{2} \mathrm{O}(\mathrm{s})$. The overall surface reaction rate is found to be limited by the adsorption rate of molecular oxygen, which depends on the concentration of molecular oxygen close to the surface of the wire and the distribution of empty sites in the catalyst. The analysis allows the determination of the critical conditions for ignition and the ignition delay time as a function of the important physicochemical parameters. The resulting wire temperatures and the critical value of the external heating rate at ignition are computed. The analysis shows how the ignition temperature increases as the external heating rate increases. The configuration dependence of the ignition temperature is taken as an indication that the use of a critical Damköhler number provides a better ignition condition than the ignition temperature concept. A self-sustained combustion regime for strong diluted mixtures is described.
\end{abstract}

\section{Introduction}

The catalytic combustion of hydrogen mixtures is of interest because it represents one of the simplest surface reaction schemes, with only a few reaction steps. It is also of practical importance, among other reasons because the catalytic removal of molecular hydrogen in nuclear plants may alleviate one of the main concerns in nuclear power safety. Typically, catalytic combustion processes have been studied either by numerical simulations using elementary chemistry [1-9] or by large activation energy asymptotic analyses using a one-step overall reaction mechanism [10-14]. Williams et al. [1] proposed a model for the catalytic combustion of hydrogen at high temperatures. These authors presented the rate parameters for the detailed surface chemistry. Warnatz and coworkers $[2,3]$ studied the catalytic combustion and ignition of hydrogen using detailed kinetic mechanisms for both surface and gas-phase reactions. Deutschmann et al. [4] studied the catalytic ignition of different fuels on different catalyst materials. In their numerical simulations, they showed that one or the other reactant almost covers the surface before ignition. In a series of experiments with very thin catalytic wires, Fassihi et al. [5] and Rinnemo et al. [6] determined the critical ignition temperature of hydrogen mixtures as a function mainly of the mixture ratio. These experiments were carried out with a low Reynolds number forced flow or with no flow at all. Other experiments by Behrendt et al. [7] and Fernandes et al. [8] addressed the critical temperature issue in a high Reynolds number stagnation flow configuration. The differences between the two sets of experimental results reveal a lack of universality of the ignition temperature concept and the need of a more profound understanding of the problem.

Treviño [15] presented an asymptotic analysis of catalytic ignition in the absence of an external energy supply, using a simplified model for the heterogeneous chemistry in a stagnation-point flow. The ignition in this case is reached by increasing the temperature of the combustible gaseous mixture. The analysis of Ref. [15] gives critical conditions for ignition that show in closed form the influence of the parameters affecting the ignition process. The results of Ref. [15] also demonstrate that ignition can be well modeled by a simple one-step global surface reaction with an activation energy that depends strongly on the dissociative character of the adsorption/desorption kinetics and with reaction orders equal to unity for the less efficiently adsorbed reactant and negative for the most efficiently adsorbed reactant. Using the same methodology, Treviño et al. 
[16] studied the autoignition of hydrogen mixtures on a thin catalytic wire under conditions of natural and forced convection. They deduced the critical conditions for ignition as represented by a critical Damköhler number instead of using a critical ambient temperature.

The objective of the present work is to extend the analysis of Ref. [16] to include an external energy input to the wire (Joule effect) and to obtain the critical conditions for ignition in a transient process. The main objective is to obtain the critical minimum external energy per unit length of wire necessary to produce ignition. Ignition is characterized by an interplay between heat generated by the surface reactions and heat loss to the ambient. The global reaction rate and the heat loss are to be obtained as functions of the physicochemical parameters involved.

\section{Formulation}

The physical problem under study is the following. A combustible mixture of $\mathrm{H}_{2}$ and $\mathrm{O}_{2}$ diluted in nitrogen, with mass fractions $Y_{\mathrm{H}_{2} \infty}$ and $Y_{\mathrm{O}_{2} \infty}$ and temperature $T_{\infty}$, is in contact with a horizontal catalytic wire of radius $a$ initially at temperature $T_{\infty}$. Starting at a time $t=0$, a certain heat, $q_{\mathrm{e}}$, per unit time and unit length of the wire is added externally by means of the Joule effect associated with the passage of an electric current through the wire. This heat increases the temperature of the wire, inducing a natural convection flow in the gas around the wire; and it may lead to ignition of the surface catalytic reactions. Assuming that the temperature of the wire is uniform and that the response of the gas phase is quasisteady, which will be justified below, the energy equation for the wire takes the form

$$
\begin{aligned}
\rho_{\mathrm{w}} c_{\mathrm{w}} \pi a^{2} \frac{d T_{\mathrm{w}}}{d t}= & q_{\mathrm{e}}+2 \pi Q a \omega \\
& -2 \pi \lambda\left(T_{\mathrm{w}}-T_{\infty}\right) N u(\operatorname{Pr}, \operatorname{R} a)
\end{aligned}
$$

where $\rho_{\mathrm{w}}$ and $c_{\mathrm{w}}$ are the density and the specific heat of the wire and $T_{\mathrm{w}}$ is its temperature. The three terms on the right-hand side of equation 1 represent the heat added externally, the heat released by the surface reactions, and the heat lost by natural convection to the surrounding gas, all given per unit time and unit length of wire. $Q$ is the heat released per mole of molecular hydrogen consumed through the global surface reaction $\mathrm{H}_{2}+1 / 2 \mathrm{O}_{2} \rightarrow \mathrm{H}_{2} \mathrm{O}$, and $\omega$ is the surface reaction rate in units of mole of molecular hydrogen consumed by unit time and unit surface of the wire. From the stoichiometry of the global reaction, the molar consumption rate of molecular oxygen is $\omega_{\mathrm{O}_{2}}=\omega / 2$, and $\omega_{\mathrm{H}_{2} \mathrm{O}}=-\omega$.

\section{Heat and Mass Transfer in the Gas}

In the third term of equation $1, \lambda$ is the thermal conductivity of the gas and $N u$ and $R a$ are the Nusselt and Rayleigh numbers, defined by

$$
\begin{aligned}
N u & =\frac{q a}{2 \pi \lambda\left(T_{\mathrm{w}}-T_{\infty}\right)} \text { and } \\
R a & =\frac{g\left(T_{\mathrm{w}}-T_{\infty}\right) a^{3}}{T_{\infty} v \alpha}
\end{aligned}
$$

where $q$ is the conduction heat flux from the wire surface to the gas, $g$ is the gravity acceleration, and $v$ and $\alpha$ are the kinematic viscosity and the thermal diffusivity of the gas. The following relationship between $N u, R a$, and the Prandtl number, $P r$, derived in Ref. [17] for Rayleigh numbers of order unity or smaller will be used in what follows:

$$
R a=\frac{1}{2 \pi P r N u} \exp \left(3 A_{0}-\frac{3}{N u}\right) F(N u, P r)
$$

Here, $A_{0}$ is a constant and $F$ is a function obtained as a correlation using numerical solutions of the steady-state governing equations. For $\mathrm{Pr}=0.72$, it was found that $A_{0}=0.96$ and $F=1+18.208 N u^{2}$ $+28.813 N u^{4}+18.254 N u^{5}$.

The mass fractions of gas-phase species at the surface of the wire, $Y_{i \mathrm{w}}$ with $i=\mathrm{H}_{2}, \mathrm{O}_{2}, \mathrm{H}_{2} \mathrm{O}$, can be expressed in terms of the surface reaction rate by solving the chemically frozen gas-phase species conservation equations. The result is

$$
Y_{i \mathrm{w}}=Y_{i \infty}-\frac{\omega_{i} L e_{i}\left(1-B_{i} N u\right) W_{i} c_{\mathrm{p}} a}{\lambda N u}
$$

where $\omega_{i}$ and $W_{i}$ are the molar consumption rate and molecular weight of species $i, c_{\mathrm{p}}$ is the specific heat of the gas at constant pressure, and $B_{i}$ is a function of the Lewis number $L e_{i}$ which was determined in Ref. [17]. For $L e_{\mathrm{O}_{2}} \approx L e_{\mathrm{H}_{2} \mathrm{O}} \approx 1$ and $L e_{\mathrm{H}_{2}} \approx 0.3$, we have $B_{\mathrm{O}_{2}}=B_{\mathrm{H}_{2} \mathrm{O}}=0$ and $B_{\mathrm{H}_{2}}=0.26$. Gasphase reactions are neglected, which is justified for low temperatures of the wire and dilute mixtures.

The characteristic time of conduction across the wire is $t_{\mathrm{w}}=a^{2} / \alpha_{\mathrm{w}}$, where $\alpha_{\mathrm{w}}$ is the thermal diffusivity of the wire. The conduction or diffusion time in the gas around the wire, which is the relevant response time of the gas when $R a=\mathrm{O}(1)$ or smaller, is $t_{0}=a^{2} / \alpha$. Finally, the characteristic time of heating of the wire for values of $q_{\mathrm{e}}$ of the order of the critical value leading to ignition is, from the balance of the left hand side and the last term of the right hand side of equation $1, t_{\mathrm{e}}=\rho_{\mathrm{w}} c_{\mathrm{w}} a^{2} / \lambda$. Here, a factor of $N u$ has been left out because the Nusselt number is never far from $\mathrm{O}(1)$ in the conditions of interest. The ratios $t_{\mathrm{w}} / t_{\mathrm{e}}$ and $t_{0} / t_{\mathrm{e}}$ are small, on the order of $10^{-3}$ when evaluated with typical values of the magnitudes involved, which justifies the assumptions of uniform wire temperature and quasi-steady gas phase used in equations 1 and 4 .

\section{Heterogeneous Reaction Model}

A model for the rate of consumption of the reactants as a function of the physicochemical parameters involved is required to complete the formulation 
TABLE 1

Heterogeneous reaction model

\begin{tabular}{lllcc}
\hline No. & \multicolumn{1}{c}{ Reaction } & $S$ & $A(\mathrm{~mol}, \mathrm{~cm}, \mathrm{~s})$ & $E(\mathrm{KJ} / \mathrm{mol})$ \\
\hline $\mathrm{la}, \mathrm{d}$ & $\mathrm{H}_{2}+2 \mathrm{Pd}(\mathrm{s}) \rightleftharpoons 2 \mathrm{H}(\mathrm{s})$ & 0.70 & $4.8 \times 10^{21}$ & $84-15 \theta_{\mathrm{H}}$ \\
$2 \mathrm{a}, \mathrm{d}$ & $\mathrm{O}_{2}+2 \mathrm{Pd}(\mathrm{s}) \rightleftharpoons 2 \mathrm{O}(\mathrm{s})$ & 0.4 & $7.1 \times 10^{21}$ & 230 \\
$3 \mathrm{r}$ & $\mathrm{H}(\mathrm{s})+\mathrm{O}(\mathrm{s}) \rightarrow \mathrm{OH}(\mathrm{s})+\mathrm{Pd}(\mathrm{s})$ & - & $6.5 \times 10^{21}$ & 11.5 \\
$4 \mathrm{r}$ & $\mathrm{H}(\mathrm{s})+\mathrm{OH}(\mathrm{s}) \rightarrow \mathrm{H}_{2} \mathrm{O}(\mathrm{s})+\mathrm{Pd}(\mathrm{s})$ & - & $6.5 \times 10^{21}$ & 17.4 \\
$5 \mathrm{r}$ & $\mathrm{OH}(\mathrm{s})+\mathrm{OH}(\mathrm{s}) \rightarrow \mathrm{H}_{2} \mathrm{O}(\mathrm{s})+\mathrm{O}(\mathrm{s})$ & - & $3.7 \times 10^{21}$ & 48.2 \\
$6 \mathrm{a}, \mathrm{d}$ & $\mathrm{H}_{2} \mathrm{O}+\mathrm{Pd}(\mathrm{s}) \rightleftharpoons \mathrm{H}_{2} \mathrm{O}(\mathrm{s})$ & 0.75 & $1.3 \times 10^{13}$ & 44 \\
\hline
\end{tabular}

of the problem. The simplified mechanism of Ref. [4] for the reaction of hydrogen and oxygen on a palladium catalyst, which is summarized in Table 1 , will be used for this purpose.

Reactions $3 r-5 r$ are surface reactions, assumed to be of the Langmuir-Hinshelwood type. Pd(s) denotes free sites on the surface of the palladium catalyst, and the concentrations of the adsorbed species are represented by their surface coverage $\theta_{i}$, defined as the ratio of the number of sites occupied by species $i$ to the total number of sites available. The rates of the surface reactions $3 \mathrm{r}-5 \mathrm{r}$ are $\omega_{3}=\mathrm{k}_{3} \theta_{\mathrm{H}} \theta_{\mathrm{O}}$, $\omega_{4}=\mathrm{k}_{4} \theta_{\mathrm{H}} \theta_{\mathrm{OH}}$, and $\omega_{5}=\mathrm{k}_{5} \theta_{\mathrm{OH}}^{2}$, respectively, in $1 / \mathrm{s}$ units, where

$$
\begin{aligned}
& k_{3}=A_{3} \Gamma \exp \left(-\frac{E_{3}}{R T}\right) \\
& k_{4}=A_{4} \Gamma \exp \left(-\frac{E_{4}}{R T}\right) \\
& k_{5}=A_{5} \Gamma \exp \left(-\frac{E_{5}}{R T}\right)
\end{aligned}
$$

with the pre-exponential factors and activation energies of Table 1 . In addition, $\Gamma \simeq 1.6603 \times$ $10^{-9} \mathrm{~mol} / \mathrm{cm}^{2}$ is the surface molar concentration of active sites and $R$ is the universal gas constant.

Reactions 1, 2, and 6 are adsorption/desorption reactions. The pre-exponential factors and the activation energies in rows 1,2 , and 6 of Table 1 apply to the desorption reactions $1 \mathrm{~d}, 2 \mathrm{~d}$, and $6 \mathrm{~d}$, which are well represented by Arrhenius kinetics. Their rates are $\omega_{1 \mathrm{~d}}=k_{1 \mathrm{~d}} \theta_{\mathrm{H}}^{2}, \omega_{2 \mathrm{~d}}=k_{2 \mathrm{~d}} \theta_{\mathrm{O}}^{2}$, and $\omega_{6 \mathrm{~d}}=$ $k_{6 \mathrm{~d}} \theta_{\mathrm{H}_{2} \mathrm{O}}$, with

$$
\begin{aligned}
& k_{1 \mathrm{~d}}=\Gamma A_{1 \mathrm{~d}} \exp \left(-\frac{E_{1 \mathrm{~d}}}{R T}\right) \\
& k_{2 \mathrm{~d}}=\Gamma A_{2 \mathrm{~d}} \exp \left(-\frac{E_{2 \mathrm{~d}}}{R T}\right) \\
& k_{6 \mathrm{~d}}=A_{6 \mathrm{~d}} \exp \left(-\frac{E_{6 \mathrm{~d}}}{R T}\right)
\end{aligned}
$$

The rates of the adsorption reactions are $\omega_{1 \mathrm{a}}=$ $k_{1 \mathrm{a}} \theta_{\mathrm{v}}^{2}, \omega_{2 \mathrm{a}}=k_{2 \mathrm{a}} \theta_{\mathrm{v}}^{2}$, and $\omega_{6 \mathrm{a}}=k_{6 \mathrm{a}} \theta_{\mathrm{v}}$, where $\theta_{\mathrm{v}}$ is the fraction of empty or vacant sites and the reaction constants can be written in terms of sticking probabilities or accommodation coefficients, $S_{i}$, which are the fractions of the collisions of species $i$ with the surface that led to successful adsorption. The rate of collisions for species $i$ is $Z_{\mathrm{w}_{\mathrm{i}}}=N_{\mathrm{A}} p_{i} / \sqrt{2 \pi W_{i} R T}$, from classical kinetic theory, where $p_{i}=p Y_{i} W / W_{i}$ is the partial pressure of species $i$ and $N_{\mathrm{A}}=6.02 \times 10^{23}$ is the Avogadro constant. Therefore,

$$
\begin{aligned}
& k_{1 \mathrm{a}}=\frac{S_{1} p Y_{\mathrm{H}_{2} \mathrm{w}} W}{\Gamma W_{\mathrm{H}_{2}}^{3 / 2} \sqrt{2 \pi R T}} \\
& k_{2 \mathrm{a}}=\frac{S_{2} p Y_{\mathrm{O}_{2} \mathrm{~W}} W}{\Gamma W_{\mathrm{O}_{2}}^{3 / 2} \sqrt{2 \pi R T}} \\
& k_{6 \mathrm{a}}=\frac{S_{6} p Y_{\mathrm{H}_{2} \mathrm{O}_{\mathrm{w}}} W}{\Gamma W_{\mathrm{H}_{2} \mathrm{O}}^{3 / 2} \sqrt{2 \pi R T}}
\end{aligned}
$$

where $W$ is the molecular weight of the mixture.

The consumption rate of molecular hydrogen is $\omega=\Gamma\left(k_{1 \mathrm{a}} \theta_{\mathrm{v}}^{2}-k_{\mathrm{ld}} \theta_{\mathrm{H}}^{2}\right)$. A similar equation can be written for the consumption rate of molecular oxygen, but here the rate of desorption of $\mathrm{O}(\mathrm{s})$ through reaction $2 \mathrm{~d}$ is extremely small compared with the rate of adsorption through reaction $2 \mathrm{a}$, so that the consumption is limited by the adsorption of molecular oxygen: $\omega_{\mathrm{O}_{2}} \approx \Gamma k_{2 \mathrm{a}} \theta_{\mathrm{v}}^{2}$. The stoichiometry of the overall reaction then requires

$$
\omega=\Gamma\left(k_{\mathrm{la}} \theta_{\mathrm{v}}^{2}-k_{1 \mathrm{~d}} \theta_{\mathrm{H}}^{2}\right) \approx 2 \Gamma k_{2 \mathrm{a}} \theta_{\mathrm{v}}^{2}
$$

which implies a relation between the surface coverage of empty sites and the surface coverage of $\mathrm{H}(\mathrm{s})$ :

$$
\theta_{\mathrm{v}} \approx K \theta_{\mathrm{H}} \quad \text { with } \quad K=\sqrt{\frac{k_{1 \mathrm{~d}}}{k_{1 \mathrm{a}}-k_{2 \mathrm{a}}}}
$$

Insofar as $\theta_{\mathrm{O}}, \theta_{\mathrm{OH}}$, and $\theta_{\mathrm{H}_{2} \mathrm{O}}$ are small compared with unity (see comments below), their contribution to the total surface coverage can be neglected and $\theta_{\mathrm{H}}+\theta_{\mathrm{v}} \approx 1$. This relation together with equation 9 determines $\theta_{\mathrm{v}} \approx K /(1+K)$ and then, from equation 8 , 


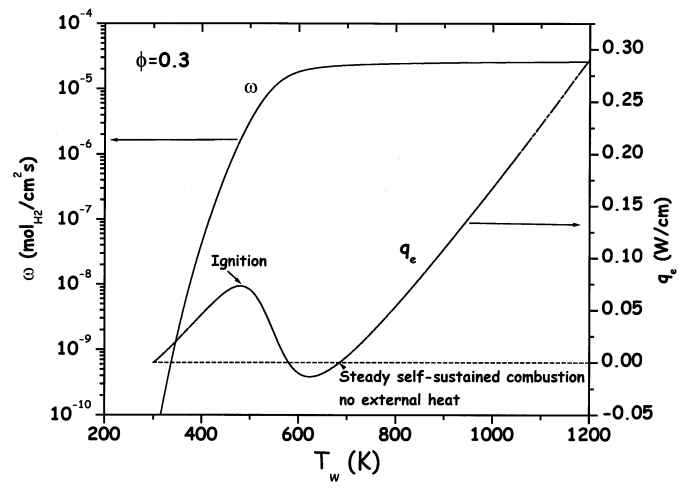

FIG. 1. External heating rate per unit length of wire and overall reaction rate as a function of wire temperature, obtained numerically for a lean but locally rich mixture $(\phi=$ $0.3)$

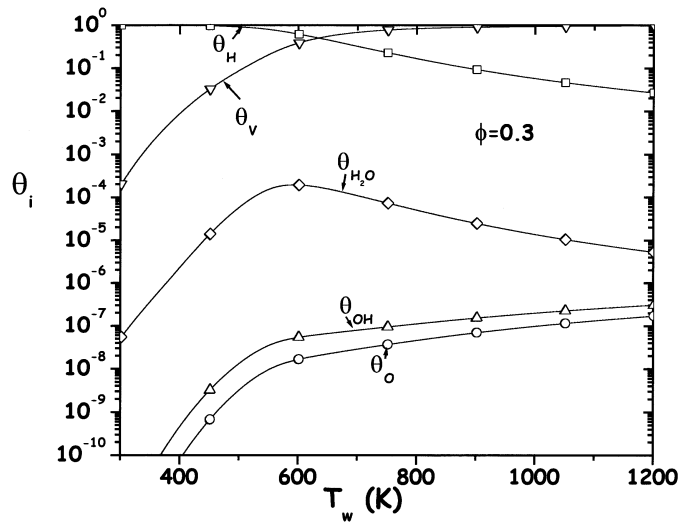

FIG. 2. Surface coverage of adsorbed species as a function of wire temperature for $\phi=0.3$.

$$
\begin{aligned}
\omega & =\frac{2 \Gamma k_{2 \mathrm{a}} K^{2}}{(1+K)^{2}} \\
& =\frac{\Gamma^{2} A_{1 \mathrm{~d}} \exp \left(-E_{1 \mathrm{~d}} / R T_{\mathrm{w}}\right)}{(1+K)^{2}\left\{32\left(S_{1} / S_{2}\right)\left(Y_{\mathrm{H}_{2} \mathrm{w}} / Y_{\mathrm{O}_{2} \mathrm{w}}\right)-1\right\}}
\end{aligned}
$$

This means that the overall surface reaction rate depends strongly on the temperature in the wire and the reactant concentrations at the surface of the wire.

\section{Further Comments}

The problem to solve consists of equations 1, 4, and 10 , with the Nusselt number given by equation 3. The elements of the formulation related to the kinetics of the surface reactions, which are summarized in equation 10, are generic insofar as the model of Ref. [4] is adopted. The elements related to the heat and mass transfer in the gas, in equations 1-4, are specific of the catalytic wire configuration. This configuration is analyzed here because of its extensive use in experiments on catalytic combustion.

Equation 4 for the two reactants $\left(i=\mathrm{H}_{2}\right.$ and $i=$ $\mathrm{O}_{2}$ ) can be rewritten as

$$
\begin{aligned}
\frac{\phi}{L e_{\mathrm{H}_{2}}\left(1-B_{\mathrm{H}_{2}} N u\right)} & \left(1-y_{\mathrm{H}_{2}}\right) \\
= & 1-y_{\mathrm{O}_{2}}=\frac{W_{\mathrm{O}_{2}}}{2 Y_{\mathrm{O}_{2}}} \frac{c_{\mathrm{p}} a \omega}{\lambda N u}
\end{aligned}
$$

where $y_{\mathrm{H}_{2}}=Y_{\mathrm{H}_{2} \mathrm{w}} / Y_{\mathrm{H}_{2} \infty}$ and $y_{\mathrm{O}_{2}}=Y_{\mathrm{O}_{2} \mathrm{w}} / Y_{\mathrm{O}_{2} \infty}$ are reduced wall mass fractions and $\phi=$ $\left(W_{\mathrm{O}_{2}} / 2 W_{\mathrm{H}_{2}}\right) Y_{\mathrm{H}_{2} \infty} / Y_{\mathrm{O}_{2} \infty}=8 Y_{\mathrm{H}_{2} \infty} / Y_{\mathrm{O}_{2} \infty}$ is the equivalence ratio, which is a measure of the richness of the mixture far from the wire. The factor in the denominator of the first term of equation 11 is a consequence of the different diffusivities of the two reactants. Its presence prompts the definition of a modified equivalence ratio $\phi_{\mathrm{r}}=$ $\phi /\left[L e_{\mathrm{H}_{2}}\left(1-B_{\mathrm{H}_{2}} \mathrm{Nu}\right)\right]$, which measures the richness of the mixture at the surface of the wire, in the sense that when $\phi_{\mathrm{r}}=1$, the first equality in equation 11 reduces to $y_{\mathrm{H}_{2}}=y_{\mathrm{O}_{2}}$ and shows that both reactants are depleted simultaneously.

The reaction rate (equation 10), with $Y_{\mathrm{H}_{2}}=$ $Y_{\mathrm{H}_{2} \infty} y_{\mathrm{H}_{2}}$ and $Y_{\mathrm{O}_{2}}=Y_{\mathrm{O}_{2} \infty} y_{\mathrm{O}_{2}}$ obtained from equation 11 , is plotted in Fig. 1 as a function of the temperature of the wire for $\phi=0.3$ and a highly diluted mixture, with 9 times more nitrogen in volume than in normal air. Also plotted in this figure is the value of the externally added heat, $q_{\mathrm{e}}\left(T_{\mathrm{w}}\right)$, that is required to keep a stationary state $\left(d T_{\mathrm{w}} / d t=0\right.$ in equation 1.) with the assumed wire temperature. Negative values of $q_{\mathrm{e}}$ mean that heat should be evacuated from the wire. The local maximum of the curve, at $q_{\mathrm{e}}=q_{\mathrm{c}}=0.07319 \mathrm{~W} / \mathrm{cm}$ in this particular case, corresponds to ignition. The middle branch, with negative slope, is a branch of unstable solutions. The rightmost root of $q_{\mathrm{e}}\left(T_{\mathrm{w}}\right)=0$, on the third branch of the curve, determines the temperature of the wire in self-sustained stationary combustion. The curve $q_{\mathrm{e}}\left(T_{\mathrm{w}}\right)$ shifts upward when the dilution is increased, until extinction occurs when the minimum of the curve gets into the region of $q_{\mathrm{e}}>0$.

Figure 2 shows the surface coverage of the adsorbed species as functions of the temperature of the wire for the same conditions of Fig. 1. These results are obtained from the equilibrium conditions

$$
\left.\begin{array}{c}
2 \omega_{1 \mathrm{a}}-2 \omega_{1 \mathrm{~d}}-\omega_{3}-\omega_{4}=0 \\
2 \omega_{2 \mathrm{a}}-2 \omega_{2 \mathrm{~d}}-\omega_{3}+\omega_{5}=0 \\
\omega_{3}-\omega_{4}-2 \omega_{5}=0 \\
\omega_{4}+\omega_{5}+\omega_{6 \mathrm{a}}-\omega_{6 \mathrm{~d}}=0 \\
\theta_{\mathrm{H}}+\theta_{\mathrm{O}}+\theta_{\mathrm{OH}}+\theta_{\mathrm{H}_{2} \mathrm{O}}+\theta_{\mathrm{V}}=1
\end{array}\right\}
$$

As was advanced before, $\theta_{\mathrm{O}}, \theta_{\mathrm{OH}}$, and $\theta_{\mathrm{H}_{2} \mathrm{O}}$ are very small compared with unity at any temperature of the wire. The dependence of $\theta_{\mathrm{H}}$ and $\theta_{\mathrm{V}}$ with $T_{\mathrm{w}}$ can be 


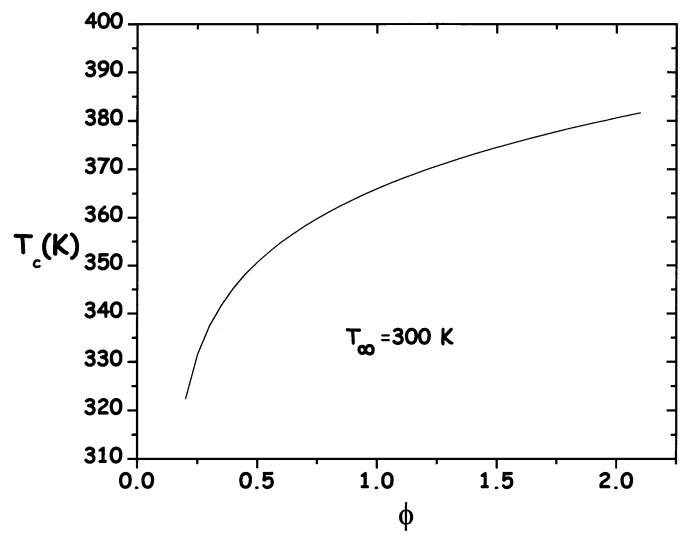

FIG. 3. Critical temperature for ignition, $T_{\mathrm{c}}$, as a function of the equivalence ratio. The considered initial temperature of the wire and mixture was $T_{\infty}=300 \mathrm{~K}$, and the palladium wire diameter was $2 a=0.0127 \mathrm{~cm}$.

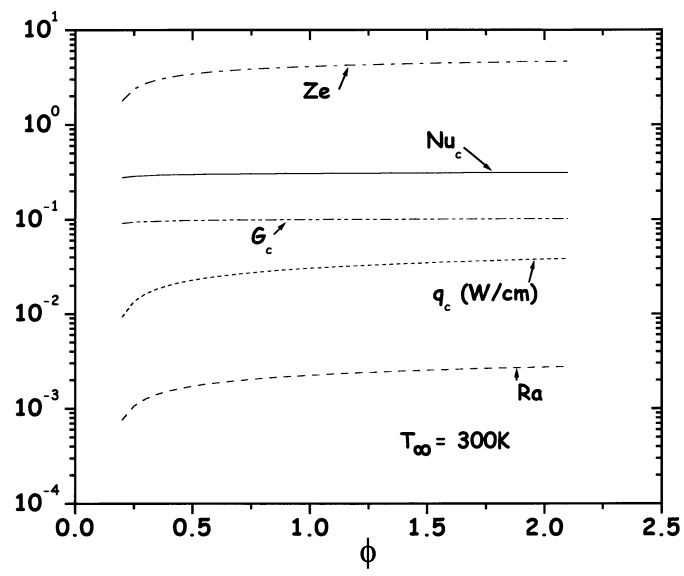

FIG. 4. Typical values of the important parameters of the ignition of hydrogen mixtures by a thin catalytic palladium wire are plotted as a function of the equivalence ratio. The non-dimensional parameters are the Zeldovich, Nusselt, and Rayleigh numbers, together with $G_{\mathrm{c}}$ introduced in equation 13. The critical external energy per unit length added to the wire, $q_{\mathrm{c}}$, is also plotted.

traced to equation 9. The large activation energy of reaction $1 \mathrm{~d}$ makes the desorption of $\mathrm{H}(\mathrm{s})$ very slow at low temperatures, so that $\theta_{\mathrm{H}} \approx 1$. The fraction of empty sites increases with temperature, and a crossover temperature exists at which $\theta_{\mathrm{H}}=\theta_{\mathrm{V}} \approx 1 / 2$ and above which the coverage of $\mathrm{H}(\mathrm{s})$ decreases rapidly and $\theta_{\mathrm{V}}$ approaches unity.

The overall reaction rate (equation 8) increases with $\theta_{\mathrm{V}}$, and therefore, in actual evolution, the nonlinear feedback due to the exothermicity of the surface reactions is prone to lead to a thermal runaway, wherein the system jumps abruptly from a nearly frozen or weakly reactive state to a state of vigorous combustion, with an important change in the wire temperature.

Radiative heat losses have been left out of the formulation. The importance of radiative losses from the wire relative to conduction losses is measured by the Boltzmann number $\sigma a T_{\mathrm{w}}^{3} / \lambda$, where $\sigma$ is the Stefan-Boltzmann constant. This number is very small, on the order of $10^{-2}$ for thin wires at the temperatures typical of ignition (say $a \sim 0.01 \mathrm{~cm}$ and $T_{\mathrm{w}}=$ $\left.T_{\mathrm{c}} \sim 340 \mathrm{~K}\right)$. Radiation may be important at the temperatures of stationary self-sustained combustion, but even then, it leads only to moderate changes of the final temperature of the wire.

\section{Ignition}

The condition $d q_{\mathrm{e}} / d T_{\mathrm{w}}=0$, at the maximum of $q_{\mathrm{e}}\left(T_{\mathrm{w}}\right)$ in Fig. 1, defines the critical temperature for ignition. With $q_{\mathrm{e}}\left(T_{\mathrm{w}}\right)$ given by the stationary form of equation 1 , this condition is $Q a d \omega / d T_{\mathrm{w}}=\lambda[N u+$ $\left.\left(T_{\mathrm{w}}-T_{\infty}\right) d N u / d T_{\mathrm{w}}\right]$. The right-hand side can be evaluated immediately using equation 2 and 3 . The result is $\lambda N u(1+G)$, with

$$
\begin{aligned}
\mathrm{G} & =\frac{d \ln N u}{d \ln R a} \\
& =\frac{1}{3 N u^{-1}-1+d \ln F / d \ln N u}
\end{aligned}
$$

The evaluation of $d \omega / d T_{\mathrm{w}}$ can be simplified using the approximations $32 Y_{\mathrm{H}_{2} \mathrm{w}} / Y_{\mathrm{O}_{2} \mathrm{w}} \approx 32 Y_{\mathrm{H}_{2} \infty} / Y_{\mathrm{O}_{2} \infty}=$ $4 \phi$ and $1+K \approx 1$ in the denominator of equation 10 , which are justified because the changes of the mass fractions of the reactants from their ambient values are small at ignition and $K$ is generally very small (except if the difference $k_{1 \mathrm{a}}-2 k_{2 \mathrm{a}}$ in equation 9 is small). With these approximations, $d \omega / d T_{\mathrm{w}}=$ $\left(E_{1 \mathrm{~d}} / R T_{\mathrm{w}}^{2}\right) \omega$ and the critical temperature is the solution of

$$
\frac{E_{1 \mathrm{~d}} Q a \omega_{\mathrm{c}}}{\lambda R T_{\mathrm{c}}^{2} N \mathrm{u}_{\mathrm{c}}}=1+G_{\mathrm{c}}
$$

where the subscript "c" denotes variables evaluated at the critical temperature $T_{\mathrm{w}}=T_{\mathrm{c}}$. The critical temperature is plotted in Fig. 3 as a function of the equivalence ratio for a wire of diameter $2 a=$ $0.0127 \mathrm{~cm}$ and $T_{\infty}=300 \mathrm{~K}$. The critical external heat $q_{\mathrm{c}}=q_{\mathrm{e}}\left(T_{\mathrm{c}}\right)$ is plotted in Fig. 4 together with some other important parameters.

The effect of the reactant consumption on the critical temperature can be accounted for by computing the values of $y_{\mathrm{H}_{2}}$ and $y_{\mathrm{O}_{2}}$ at ignition from equation 11 with $\omega=\omega_{\text {c }}$ given by equation 14 . The result is $\left(y_{\mathrm{H}_{2}}\right)_{\mathrm{ig}}=1-\beta$ and $\left(y_{\mathrm{O}_{2}}\right)_{\mathrm{ig}}=1-\phi_{\mathrm{r}} \beta$, with 


$$
\beta=\left(1+G_{\mathrm{c}}\right) \frac{\phi}{\phi_{\mathrm{r}}} \frac{W_{\mathrm{H}_{2}} c_{\mathrm{p}} T_{\mathrm{c}}}{Q Y_{\mathrm{H}_{2} \infty}} \frac{R T_{\mathrm{c}}}{E_{1 \mathrm{~d}}}
$$

which is generally very small compared with unity. Using these reduced mass fractions and taking advantage of the smallness of $\beta$, the effect of the reactant consumption on the critical temperature can be evaluated as

$$
\begin{aligned}
& T_{\mathrm{c}}=T_{\mathrm{c} 0} \\
& \times\left[1+\frac{\beta \frac{4 \phi S_{1}}{S_{2}}\left(\phi_{\mathrm{r}}-1\right)}{\left(\frac{4 \phi S_{1}}{S_{2}}-1\right)\left(\frac{E_{1 \mathrm{~d}}}{R T_{\mathrm{c} 0}}-\frac{G_{\mathrm{c}} T_{\mathrm{c} 0}}{T_{\mathrm{c} 0}-T_{\infty}}-2\right)}+\mathrm{O}\left(\beta^{2}\right)\right]
\end{aligned}
$$

where $T_{\mathrm{c} 0}$ is the critical temperature with no reactant consumption. As expected, for local rich mixtures, the critical temperature increases with reactant consumption, while for local lean mixtures the critical temperature decreases. For local stoichiometric mixtures, $\phi_{\mathrm{r}}=1$, reactant consumption does not have any influence on the ignition process.

The time evolution of the temperature of the wire during ignition is also of interest. In terms of the non-dimensional variables and parameters

$$
\begin{aligned}
& \Theta=\frac{T_{\mathrm{w}}-T_{\infty}}{T_{\mathrm{c}}-T_{\infty}}, \quad \tau=\frac{\lambda N u_{\mathrm{c}} t}{\rho_{\mathrm{w}} c_{\mathrm{w}} a^{2}} \\
& \gamma=\frac{q_{\mathrm{e}}}{2 \pi \lambda\left(T_{\mathrm{c}}-t_{\infty}\right) N u_{\mathrm{c}}}, Z e=\frac{E_{1 \mathrm{~d}}\left(T_{\mathrm{c}}-T_{\infty}\right)}{R T_{\mathrm{c}}^{2}}
\end{aligned}
$$

equation 1 takes the form

$$
\begin{aligned}
\frac{d \Theta}{d \tau}= & \gamma-\Theta \frac{N u}{N u_{\mathrm{c}}}+\frac{1+G_{\mathrm{c}}}{Z e} \\
& \times \exp \left[\frac{Z e(\Theta-1)}{1+\frac{T_{\mathrm{c}}-T_{\infty}}{T_{\mathrm{c}}}(\Theta-1)}\right]
\end{aligned}
$$

to be solved with the initial condition $\Theta(0)=0$. The Zeldovich number $Z e$ evaluated with the activation energy $E_{1 d}$ of Table 1 and the critical temperatures of Fig. 3 is always very large, which makes the reaction term negligible in equation 17 for any $(1-$ $\Theta) \gg Z e^{-1}$. The temperature coincides then with the solution of

$$
\frac{d \Theta_{\mathrm{f}}}{d \tau}=\gamma-\Theta_{\mathrm{f}} \frac{N u}{N u_{\mathrm{c}}}, \quad \Theta_{\mathrm{f}}(0)=0
$$

which is straightforward. The reaction term comes into play when $\Theta$ gets into the range $(1-\Theta)=$ $\mathrm{O}\left(Z e^{-1}\right) \ll 1$, where the exponent of equation 17 can be linearized and the Nusselt number can be replaced by the first term of its Taylor expansion about $N u_{\mathrm{c}}$. With these simplifications, equation 17 becomes

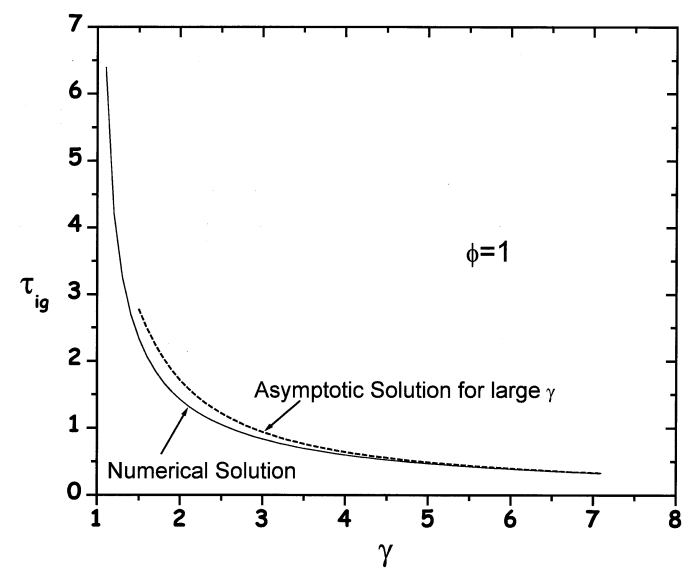

FIG. 5. Non-dimensional ignition delay time as a function of the parameter $\gamma$ for a stoichiometric mixture of hydrogen/oxygen. The asymptotic solution for large values of $\gamma$ is also plotted.

$$
\begin{aligned}
\frac{d \Theta}{d \tau}= & \gamma-\left(1-G_{\mathrm{c}}\right) \Theta-G_{\mathrm{c}} \Theta^{2} \\
& +\frac{1+G_{\mathrm{c}}}{Z e} \exp [\operatorname{Ze}(\Theta-1)]
\end{aligned}
$$

Two stationary solutions of equation 19 exist for $\gamma<$ $\gamma_{\mathrm{c}}=1-\left(1+G_{\mathrm{c}}\right) / \mathrm{Ze}$ (which is a non-dimensional form of $q_{\mathrm{c}}$ ), while $\Theta$ diverges in a finite ignition time for $\gamma>\gamma_{\mathrm{c}}$. The ignition time can be easily computed when $\left(\gamma-\gamma_{\mathrm{c}}\right) \gg Z e^{-1}$. Let $\tau_{\mathrm{I}}$ denote this time, $\Theta_{\mathrm{I}}=\Theta_{\mathrm{f}}\left(\tau_{\mathrm{I}}\right)$ be the frozen temperature determined by the solution of equation 18 at the ignition time, and $\Theta_{\mathrm{I}}^{\prime}=d \Theta_{\mathrm{f}} /\left.d \tau\right|_{\tau \mathrm{I}}=\gamma-\left(1-G_{\mathrm{c}}\right) \Theta_{\mathrm{I}}-G_{\mathrm{c}} \Theta_{\mathrm{I}}^{2}$. In terms of the new variables,

$$
\begin{aligned}
\psi & =Z e\left(\Theta-\Theta_{\mathrm{f}}\right), \sigma=Z_{e} \Theta_{\mathrm{I}}^{\prime}\left(\tau-\tau_{\mathrm{I}}\right), \\
\delta & =\frac{1+G_{\mathrm{c}}}{Z e \Theta_{\mathrm{I}}^{\prime}} \exp \left[\mathrm{Ze}\left(\Theta_{\mathrm{I}}-1\right)\right]
\end{aligned}
$$

equation 19 becomes $d \psi / d \sigma=\delta \exp (\psi+\sigma)$ up to a small term of $\mathrm{O}(\psi / \mathrm{Ze})$. The solution of this equation with $\psi(-\infty)=0$ should diverge when $\sigma \nearrow 0$, which happens for $\delta=1$. This condition determines $\Theta_{\mathrm{I}}$ and therefore the ignition time $\tau_{\mathrm{I}}$. Both variables are plotted in Figs. 5 and 6 as functions of $\gamma$. As can be seen, the ignition time tends to infinity when $\gamma \searrow \gamma_{\mathrm{c}}$ and has the asymptotic behavior $\tau_{\mathrm{I}}=$ $\Theta_{\mathrm{I}} / \Theta_{\mathrm{I}}^{\prime} \approx 1 / \gamma$ (dashed curve in Fig. 5) for large values of $\gamma$. The linearization of the Nusselt number used in equation 19, which is justified for small values of $\Theta-1$, proves to be a good approximation in the whole range of $\Theta$ when it is used in equation 18. This equation has then a simple analytical solution that has been used to compute $\tau_{\mathrm{I}}$ in Fig. 5 . 


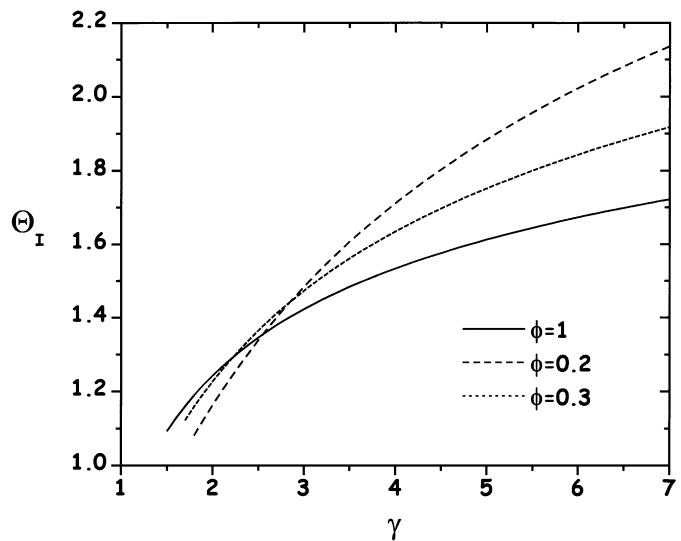

FIG. 6. Non-dimensional wire temperatures at ignition as a function of $\gamma$, for three different equivalence ratios.

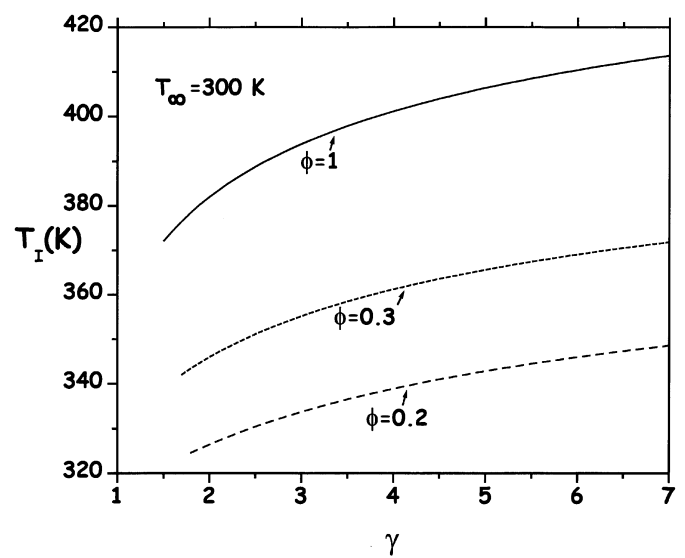

FIG. 7. Wire temperatures at ignition as a function of $\gamma$, for three different equivalence ratios.

\section{Self-Sustained Combustion}

The temperature of the wire in stationary self-sustained combustion is often sufficiently higher than the crossover temperature introduced in the paragraph following equation 12 that $K \gg 1$ and the approximation $\theta_{\mathrm{V}} \approx 1$ can be used in the rightmost term of equation 8 . Then, the second equality in equation 11 gives

$$
\frac{\omega}{2} \approx \Gamma k_{2 \mathrm{a} \infty} y_{\mathrm{O}_{2}}=\left(1-y_{\mathrm{O}_{2}}\right) \frac{\lambda N u Y_{\mathrm{O}_{2} \infty}}{W_{\mathrm{O}_{2}} c_{\mathrm{p}} a}
$$

where $k_{2 \mathrm{a} \infty}=k_{2 \mathrm{a}}\left(Y_{\mathrm{O}_{2} \infty}\right)$. From here,

$$
y_{\mathrm{O}_{2}} \approx\left[1+\frac{W_{\mathrm{O}_{2}} c_{\mathrm{p}} a \Gamma k_{2 \mathrm{a} \infty}}{\lambda Y_{\mathrm{O}_{2} \infty} N u}\right]^{-1}
$$

On the other hand, eliminating $\omega$ between equation 1 , with $d T_{\mathrm{w}} / d t=q_{\mathrm{e}}=0$, and equation 4 for $i=$ $\mathrm{O}_{2}$, gives

$$
T_{\mathrm{w}}=T_{\infty}+\left(1-y_{\mathrm{O}_{2}}\right) \frac{2 Y_{\mathrm{O}_{2} \infty} Q}{W_{\mathrm{O}_{2}} c_{\mathrm{p}}}
$$

which determines the temperature of the wire when $y_{\mathrm{O}_{2}}$ is eliminated with the help of equation 20.

\section{Conclusions}

Numerical and asymptotic techniques have been used in this work to describe the transient ignition and combustion of a diluted mixture of hydrogen and air using an externally heated thin palladium catalytic wire. There is a critical external thermal energy per unit length of the wire below which no catalytic ignition is possible. Using this critical external energy, ignition is achieved for a wire temperature which increases with the equivalence ratio as shown in Fig. 3, in agreement with previously reported experimental results $[18,19]$. However, if the external energy input is increased to values larger than the critical one, the resulting wire temperature at ignition is not only a function of the equivalence ratio of the mixture but also of the parameter $\gamma$, which measures the departure from the critical external energy input. Therefore, for a given parametric set, including the ambient temperature and diameter of the wire, the wire temperature at ignition increases also as the external heat input also increases. Fig. 7 shows this wire temperature at ignition as a function of $\gamma$, for three different values of the equivalence ratio. This is a clear indication that the temperature at ignition is not unique and depends on how the ignition conditions are reached. A better indication of the ignition conditions should be given by a Damköhler defined as in the left-hand side of equation 14.

$$
\text { Acknowledgment }
$$

This work has been supported by CONACyT, Mexico, under grant 33349-U.

\section{REFERENCES}

1. Williams, W. R., Marks, C. M., and Schmidt, L. D., J. Phys. Chem. 96:5922 (1992).

2. Warnatz, J., Proc. Combust. Inst. 24:533 (1992).

3. Warnatz, J., Allendorf, M. D., Kee, R. J., and Coltrin, M., Combust. Flame 96:393 (1994).

4. Deutschmann, O., Schmidt, R., Behrendt, F., and Warnatz, J., Proc. Combust. Inst. 26:1747 (1996).

5. Fassihi, M., Zhdanov, V. P., Rinnemo, M., Keck, K.-E., and Kasemo, B., J. Catal. 141:438-452 (1993).

6. Rinnemo, M., Deutschmann, O., Behrendt, F., and Kasemo, B., Combust. Flame 111:312-326 (1997).

7. Behrendt, F., Deutschmann, O., Schmidt, R., and Warnatz, J., "Ignition and Extinction of Hydrogen-Air and Methane-Air Mixtures over Platinum and Palladium," in Heterogeneous Hydrocarbon Oxidation 
(B. K. Warren and S. T. Oyama, eds.), ACS Symposium Series, No. 68, Oxford, UK, 1996.

8. Fernandes, N. E., Park, Y. K., and Vlachos, D. G., Combust. Flame 118:164-178 (1999).

9. Bui, P.-A., Vlachos, D. G., and Westmoreland, P. R., Proc. Combust. Inst. 26:1763 (1996).

10. Treviño, C., and Fernández-Pello, A. C., Combust. Sci. Technol. 26:245 (1981).

11. Treviño, C., Combust. Sci. Technol. 30:213 (1983).

12. Treviño, C., and Peters, N., Combust. Flame 61:39 (1985).

13. Song, X., Williams, W. R., Schmidt, L. D., and Aris, R., Proc. Combust. Inst. 23:1129 (1990).
14. Treviño, C., and Méndez, F., Proc. Combust. Inst. 26:1797 (1996).

15. Treviño, C., Combust. Theory Modelling 3:469-477 (1999).

16. Treviño, C., Liñán, A., and Kurdyumov, V., Proc. Combust. Inst. 28:1359-1364 (2000).

17. Liñán, A., and Kurdyumov V., J. Fluid Mech. 362:199 227 (1998).

18. Rinnemo, M., Kulginov, D., Johansson, S., Wong, K. L., Zhdanov, V. P., and Kasemo, B., Surf. Sci. 376:297 (1997).

19. Cho, P., and Law C. K., Combust. Flame 66:159 (1986). 